\title{
Determination of chlorpheniramine enantiomers in pharmaceutical formulations by HPLC on chiral column with PDA detection
}

\author{
Gizem Erensoy ${ }^{1,2}$ (D) Duygu Tașkın³,4 (D), Gamze Özgül Artuç ${ }^{1}$ (D), Elif Özdemir ${ }^{3}$ (D), Sumru Özkırımlı ${ }^{10}$ \\ 'İstanbul Yeni Yüzyıl University, Faculty of Pharmacy, Department of Pharmaceutical Chemistry, Istanbul, Turkey \\ ${ }^{2}$ Marmara University, Faculty of Pharmacy, Department of Pharmaceutical Chemistry, Istanbul, Turkey \\ ${ }^{3}$ Istanbul Yeni Yüzyıl University, Faculty of Pharmacy, Department of Analytical Chemistry, Istanbul, Turkey \\ ${ }^{U}$ University of Health Sciences, Faculty of Pharmacy, Department of Analytical Chemistry, Istanbul, Turkey
}

ORCID IDs of the authors: G.E. 0000-0001-5991-4833; D.T. 0000-0002-5279-0900; G.0̈.A. 0000-0002-7869-1281;

E.Ö. 0000-0002-5011-8344; S.0̈. 0000-0002-7939-2750

Cite this article as: Erensoy, G., Taskin, D., Ozgul Artuc, G., Ozdemir, E., \& Ozkirimli, S. (2020). Determination of chlorpheniramine enantiomers in pharmaceutical formulations by HPLC on chiral column with PDA detection. Istanbul Journal of Pharmacy, 50 (2), 149-152.

\begin{abstract}
Background and Aims: An HPLC method with photodiode array detector on a chiral column was proposed for enantioselective determination of chlorpheniramine (CLP) enantiomers in dosage forms.

Methods: The enantioselective determination was achieved on amylose tris(3,5-dimethylphenylcarbamate) column, using $\mathrm{n}$-hexane-(propan-2-ol)-diethylamine (97.5:2.5:0.025, v/v/v) mobile phase. The peaks were detected at $258 \mathrm{~nm}$. Diphenhydramine was used as an internal standard (IS). A new sample preparation procedure was developed to avoid the interference of the other ingredients present in the formulations.

Results: Limit of quantification of the proposed method was 0.88 and $1.31 \mu \mathrm{g} / \mathrm{mL}$ for S-(+)-CLP and R-(-)-CLP, respectively. Conclusion: The method is linear, sensitive, specific and can be used for the enantioselective assay of CLP enantiomers in pharmaceutical formulations.

Keywords: Chlorpheniramine, amylose tris(3,5-dimethyl phenylcarbamate), enantioselective determination, HPLC-PDA, chirality
\end{abstract}

\section{INTRODUCTION}

Stereochemistry of drugs is an important topic for the pharmaceutical industry and the regulatory authorities because enantiomers of chiral drugs may exhibit different biological activities with only one of the enantiomers exhibiting therapeutic value while the others are less effective or toxic. There has been increasing interest in the stereospecific analysis of chiral drug molecules (Calcaterra \& D'Acquarica 2018).

Chlorpheniramine (CLP), 3-(4-chlorophenyl)-N,N-dimethyl-3-(pyridin-2-yl)propan-1-amine, is a first generation histamine H1 receptor antagonist (Figure 1).

CLP is mostly marketed as a racemate, only a few dosage forms are available as a single S-(+)-CLP. In tissues, the S-(+) enantiomer of chlorpheniramine (dexchlorpheniramine) has a 13-fold greater affinity than its $\mathrm{R}-(-)$ enantiomer to $\mathrm{H} 1$ receptors (Tanda, Kopajtic, 


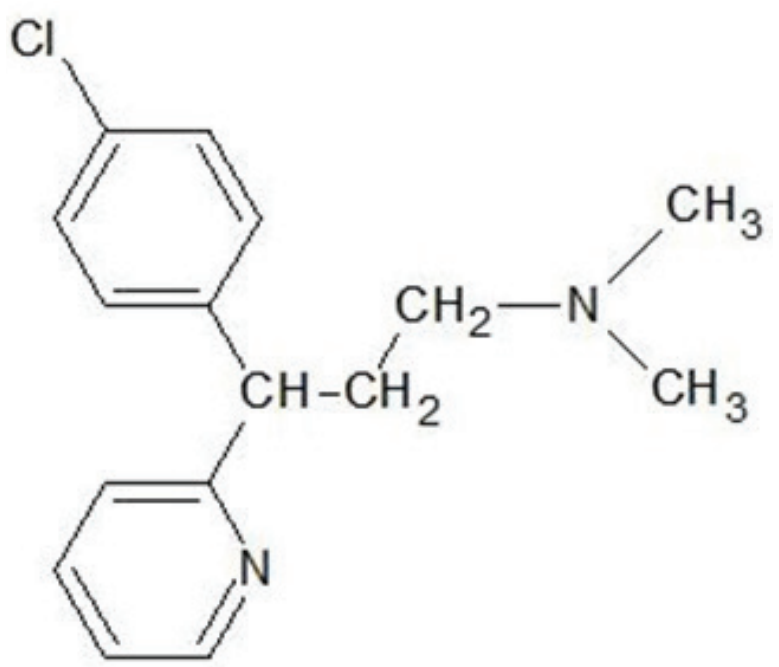

Figure 1. Chemical structure of chlorpheniramine.

\& Katz, 2008). S-(+)-CLP has antihistaminic activity and R-(-)-CLP exhibits sedative side effects (Tagawa et al., 2002).

Studies on the enantioselective determination of rac-CLP have been limited. Studies for stereoselective determination have used $\beta$-cyclodextrin chiral stationary phase with mass spectrometric detection (Fried, Young, Yasuda, \& Wainer, 2001), coupled achiral (cyanopropyl)-chiral (Amylose) stationary phase with UV detection (Hiep, Khanh, Hung, Thuillier, \& Gimenez, 1998), or ODS column with $\beta$-cyclodextrin as a mobile phase additive (Chen, Jeong, Hwang, Kim, \& Kang, 2008).

Here, we describe an enantioselective determination of CLP enantiomers in syrup containing a high dose of paracetamol by HPLC on an amylose column with PDA detection. The proposed novel method was validated according to ICH guidelines in terms of precision, linearity and accuracy.

\section{MATERIALS AND METHODS}

\section{Instrumentation}

The HPLC system consisted of LC-20AT liquid system (Shimadzu Corporation Analytical System) equipped with a degasser (DGU-20A5R), photodiode array detector (SPD-M20A PDA) and a rheodyne syringe sample injector $(50 \mu \mathrm{L})$. The enantioselective separation of S-(+) and R-(-) enantiomers was performed on a Chiralpak AD-H column (250 mm x $4.6 \mathrm{~mm}$ i.d.) with $5 \mu \mathrm{m}$ particle size (Daicel).

The mobile phase consisted of a mixture of n-hexane-(propan2-ol, IPA)-diethylamine (DEA) (97.5:2.5:0.025, v/v/v). Flow rate was $1.2 \mathrm{~mL} / \mathrm{min}$ at isocratic mode. The eluents were monitored at $258 \mathrm{~nm}$. All analyses were performed at $25^{\circ} \mathrm{C}$.

\section{Chemicals}

Racemic chlorpheniramine maleate (rac-CLP-M) and diphenhydramine hydrochloride (DPH) were kindly supplied by Bilim Illaç A.Ş. (Istanbul, Turkey). S-(+)CLP-M was obtained from Kiwidrug (New Zealand). n-Hexane (HPLC grade), propan-2-ol (HPLC grade), were purchased from Merck (Darmstadt, Germany). Diethylamine (DEA) was obtained from Fluka (Switzerland).

\section{Preparation of stock solutions}

The stock solutions of rac-CLP-M $(400 \mu \mathrm{g} / \mathrm{mL}$, calculated as free base) and DPH (IS) (1 mg/mL) were prepared in distilled water. Quality control samples containing 2, 4, 6, 8, and $10 \mu \mathrm{g} / \mathrm{mL}$ of corresponding enantiomers of CLP were prepared by diluting the rac-CLP-M stock solution with distilled water. All solutions were stored at $4^{\circ} \mathrm{C}$.

\section{Sample preparation procedure}

$1 \mathrm{~mL}$ aliquots of quality control samples, $25 \mu \mathrm{L}$ of IS and 100 $\mu \mathrm{L}$ of $0.1 \mathrm{M} \mathrm{NaOH}$ solutions were placed in a $15 \mathrm{~mL}$ conical glass centrifuge tube. The samples were extracted with $1.5 \mathrm{~mL}$ of $\mathrm{n}$-hexane-dichloromethane $(2: 1 \mathrm{v} / \mathrm{v})$ by vortex-mixing for 2 min and centrifuged at 2500 rpm for 10 minutes. $1 \mathrm{~mL}$ of the organic phase was separated and evaporated to dryness under a gentle stream of nitrogen and reconstituted in $300 \mu \mathrm{L}$ of mobile phase. A $50 \mu \mathrm{L}$ aliquot of the solution was injected into the chromatographic system.

\section{Assay of pharmaceutical dosage forms}

$1 \mathrm{~mL}$ aliquot of commercial syrup (160 mg paracetamol and $1 \mathrm{mg}$ rac-CLP-M in $5 \mathrm{~mL}$ of syrup) was diluted to $10 \mathrm{~mL}$ with distilled water, sonicated and $1 \mathrm{~mL}$ aliquot of the solution was used for analysis according to the sample preparation procedure. The concentration of CLP enantiomers in syrup was calculated using the regression equation $(n=6)$.

\section{Method validation}

The developed method was validated according to ICH guidelines (ICH Guideline, Q2(R1), 2005). Calibration lines were constructed by plotting the peak area ratio (PAR) against the corresponding concentration of enantiomers. Limit of quantification (LOQ) and limit of detection (LOD) were determined using $10 \mathrm{\sigma} / \mathrm{s}$ and $3.3 \mathrm{\sigma} / \mathrm{s}$, respectively. Intra- and interday precision was determined by performing four consecutive injections at three concentration levels $(4,6,8 \mu \mathrm{g} / \mathrm{mL})$ of S-(+)- and R-(-)-CLP enantiomers. Accuracy of the method was determined by adding standard CLP solutions at $8.5 \mu \mathrm{g} / \mathrm{mL}$ and 5.5 $\mu \mathrm{g} / \mathrm{mL}$ levels to syrup samples.

\section{RESULTS AND DISCUSSION}

\section{Method development}

Effects of modifier and solvent composition on retention and separation of enantiomers were investigated. n-Hexane-IPADEA (97.5:2.5:0.025, v/v/v) provided the best results for the separation of IS and CLP enantiomers.

A representative chromatogram of CLP enantiomers under optimum conditions is shown in Figure 2. Baseline separation was achieved for enantiomers with a value of 1.24. Peak resolution value was 3.80. S-(+)-CLP was used to identify the peaks of the CLP enantiomers.

The total run time of the analysis was $15 \mathrm{~min}$. The average retention time and standard deviation of ten replicates were $9.63 \pm 0.05$ min and $11.36 \pm 0.08$ min for S-(+) and R-(-) enantiomers, respectively. No interfering peaks were observed at the same retention times of IS, S-(+)-CLP and R-(-)-CLP, and confirmed the specificity of the developed method. 


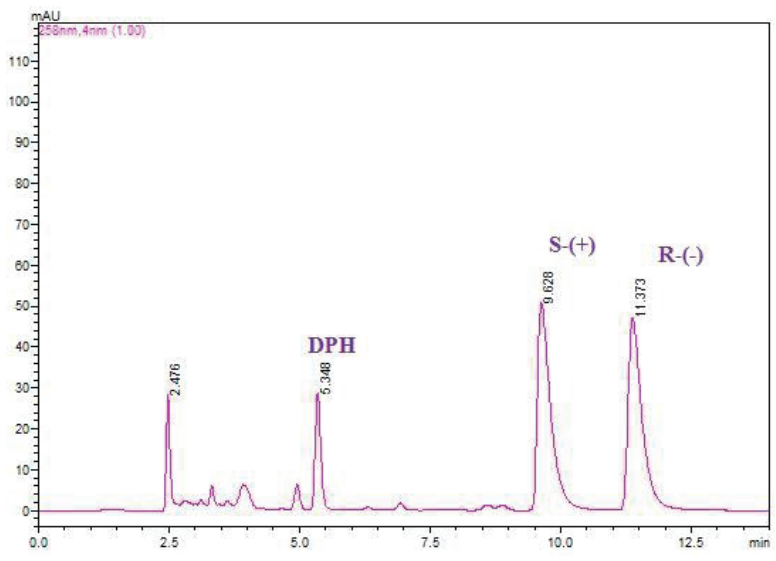

Figure 2. Representative chromatogram of CLP enantiomers and DPH (IS) on amylose tris(3,5-dimethyl phenylcarbamate) column. Conditions: mobile phase: n-Hexane-IPA-DEA (97.5:2.5:0.025 v/v/v) at $1.2 \mathrm{~mL} / \mathrm{min}$ flow rate; detection: $258 \mathrm{~nm}$.

\section{Assay validation}

The method was found linear within the range $2-10 \mu \mathrm{g} / \mathrm{mL}$ with a correlation coefficient ( $r$ ) of 0.999 for both S-(+)- and R-(-)-CLP enantiomers. The regression equations were found to be $y=0.4175( \pm 0.17) x-0.017( \pm 0.007)$ and $y=0.4195( \pm 0.17)$ $\mathrm{x}-0.011$ ( \pm 0.005$)$ for $\mathrm{S}-(+)$ - and $\mathrm{R}-(-)-C L P$, respectively.

The limit of detection (LOD) and limit of quantification (LOQ) of the CLP enantiomers were determined using calibration standards. LOD of the proposed method was 0.29 and $0.44 \mu \mathrm{g} / \mathrm{mL}$ for S-(+) and R-(-)-CLP, respectively. LOQ of the proposed method was 0.88 and $1.31 \mu \mathrm{g} / \mathrm{mL}$ for $\mathrm{S}-(+)$ and $\mathrm{R}-(-)-C L P$, respectively.

Intraday and interday precision values of the method were determined by analysing the samples on the same day and on three different days at three different concentrations for each analyte $(n=4)$. Precision of the method was expressed by relative standard deviation (RSD \%). Interday precision values were found in the range of (RSD \%) 0.24-0.61 and 1.28-1.40 for S(+) and $\mathrm{R}(-)$ enantiomers, respectively. Intraday precision values were found in the range of (RSD \%) 0.25-1.40 and 1.34-1.50 for $\mathrm{S}-(+)$ and $\mathrm{R}-(-)$ enantiomers, respectively.

The accuracy of the method was evaluated by spiking the syrup formulation with standard rac-CLP $-\mathrm{M}$ solution. The mean percent recovery (RSD \%) values were found as 99.41 (0.04) and 99.64 (0.04) at $8.5 \mu \mathrm{g} / \mathrm{mL}$ level for S-(+) and R-(-) enantiomers, respectively. RSD \% values were found as 99.64 (0.06) and $101.82(0.02)$ at $5.5 \mu \mathrm{g} / \mathrm{mL}$ level for S-(+) and R-(-) enantiomers, respectively.

\section{Analysis of commercial syrup}

CLP enantiomers in two dosage forms were analysed according to the validated method. Analyzed commercial syrups contain high doses of paracetamol $(160 \mathrm{mg} / 5 \mathrm{~mL}$ ) compared to rac-CLP-M (1 mg/5 mL). Paracetamol is insoluble in nonpolar- and chlorohydrocarbons (Granberg \& Rasmuson, 1999).
Different extraction solvents were tested. Using the n-Hexanedichloromethane (2:1) mixture allowed selective extraction of CLP enantiomers from syrup without any interference of ingredients in the formulation.

The content of S-(+)-CLP and R-(-)-CLP enantiomers in the syrup were found as (mean \% \pm SD) $99.2 \pm 0.09$ and $97.8 \pm 0.07$ for batch $1 ; 98.2 \pm 0.08$ and $98.0 \pm 0.09$ for batch 2 respectively.

\section{CONCLUSION}

In this study, we propose a novel, simple, and rapid chiral HPLC method for the determination of CLP enantiomers in formulations containing high concentration of paracetamol. In this novel extraction procedure, the use of the non-polar solvent system $\mathrm{n}$-hexane-dichloromethane (2:1) provides selectivity, and the use of diphenhydramine as an internal standard allows sensitive, precise and linear enantiomer determination.

Peer-review: Externally peer-reviewed.

Author Contributions: Conception/Design of Study- G.E., D.T., G.Ö.A., E.Ö., S.Ö.; Data Acquisition- G.E., D.T., G.Ö.A., E.Ö., S.Ö.; Data Analysis/Interpretation- G.E., D.T., G.Ö.A., E.Ö., S.Ö.; Drafting Manuscript- G.E., D.T., G.Ö.A., E.Ö., S.Ö.; Critical Revision of Manuscript- G.E., D.T., G.Ö.A., E.Ö., S.Ö.; Final Approval and Accountability- G.E., D.T., G.Ö.A., E.Ö., S.Ö.; Technical or Material Support- S.Ö.; Supervision- S.Ö.

Conflict of Interest: The authors have no conflict of interest to declare.

Financial Disclosure: Authors declared no financial support.

\section{REFERENCES}

- Calcaterra, A., \& D'Acquarica I. (2018). The market of chiral drugs: Chiral switches versus de novo enantiomerically pure compounds Journal of Pharmaceutical and Biomedical Analysis, 147, 323-340.

- $\quad$ Chen, Q., Jeong, S., Hwang, G., Kim, K., \& Kang, J. (2008). Enantioselective determination of chlorpheniramine in various formulations by HPLC using carboxymethyl- $\beta$-cyclodextrin as a chiral additive. Archives of pharmacal research, 31, 523-529.

- $\quad$ Fried, K. M., Young, A. E., Yasuda, U. S., \&Wainer, I. W. (2001). The enantioselective determination of chlorpheniramine and its major metabolites in human plasma using chiral chromatography on a $\beta$-cyclodextrin chiral stationary phase and mass spectrometric detection. Journal of Pharmaceutical and Biomedical Analysis, 27, 479-488.

- Granberg, R. A., \& Rasmuson, A. C. (1999). Solubility of paracetamol in pure solvents. Journal of Chemical \& Engineering Data, 44, 1391 1395.

- Hiep, B. T., Khanh, V., Hung, N. K., Thuillier, A., \& Gimenez, F. (1998). Determination of enantiomers of chlorpheniramine and its main monodesmethyl metabolite in urine using achiral-chiral liquid chromatography. Journal of Chromatography B. 707, 235-240. International Conference on Harmonization. ICH. 2005. Validation of Analytical Procedures: Text and Methodology Q2 R1. Geneva: International Conference on Harmonization. Available from: https://www.gmp-compliance.org/guidemgr/files/Q2(R1).pdf 
Istanbul J Pharm 50 (2): 149-152

- $\quad$ Tagawa, M., Kano, M., Okamura, N., Higuchi, M., Matsuda, M., Mizuki, Y., Yanai, K. (2002). Differential cognitive effects of ebastine and (+)-chlorpheniramine in healthy subjects: Correlation between cognitive impairment and plasma drug concentration. British Journal of Clinical Pharmacology, 53, 296-304.
- $\quad$ Tanda, G., Kopajtic, T. A., \& Katz, J. L. (2008). Cocaine-like neurochemical effects of antihistaminic medications. Journal of NeuroChemistry, 106, 147-157. 